\title{
On the relationship between internal porosity and the tensile ductility of aluminium alloy die-castings
}

\author{
Ewan Lordan ${ }^{1 *}$, Jaime Lazaro-Nebreda ${ }^{1}$, Yijie Zhang ${ }^{1}$, Kun Dou ${ }^{1}$ \\ Paul Blake ${ }^{2}$, and Zhongyun Fan ${ }^{1}$ \\ 1.-Brunel Centre for Advanced Solidification Technology (BCAST) Brunel University, \\ Uxbridge, Middlesex, UB83PH, United Kingdom. 2.- Jaguar Land Rover Research, \\ International Development Lab, Warwick University, United Kingdom.
}

\author{
* Corresponding author (E. Lordan) \\ Email: ewan.lordan@brunel.ac.uk,ewan-lordan@live.co.uk
}

\section{Abstract}

This paper describes the application of X-ray tomography to predict the tensile fracture properties of aluminium alloy die-castings containing natural casting defects. A new estimator (Z-project) is presented that can predict the areal fraction of porosity involved during tensile failure to a higher degree of accuracy than conventional methods, which are also discussed. By coupling the proposed estimator with an existing model for the development of plastic instabilities in a deforming body, the tensile fracture strain and tensile fracture stress are predicted with much less error than previous approaches.

Keywords: Defects, X-ray tomography, Mechanical properties, Aluminium alloys, Die casting.

\section{Introduction}

Cast aluminium alloys are finding increasing applications in lightweight vehicle architecture, due to their competitive specific strength and excellent castability. For the purpose of cost reduction and part consolidation, die-castings typically exhibit a high degree of geometric complexity, which results in a certain amount of porosity that is practically unavoidable in normal industrial castings [1-3]. As the dominant ductility limiting factor in hypoeutectic Al-Si$\mathrm{Mg}$ alloys, porosity presents a major obstacle to the more widespread use of aluminium castings within the automotive industry, often responsible for the conservative safety coefficients used in component design [4]. To address this issue, further understanding is required before a quantitative relationship may be established between the casting integrity and the materials performance in tensile and fatigue.

By performing 3D in-situ tensile and fatigue tests using X-ray tomography and digital volume correlation, several studies have revealed the damage mechanisms of hypoeutectic Al-Si alloys [4-13]. It is widely accepted, that damage originates at various microstructural inhomogenieties including eutectic Si particles and intermetallic phases. The nucleation of voids from these hard inclusions occurs by decohesion of the particle-matrix interface or by particle fracture, with the subsequent growth and coalescence of voids forming large cracks in the Al matrix [14]. By reducing the load-bearing area and localising strain, pores provide 
preferential crack initiation sites and accelerate the production of damage $[1,2,10]$. Whilst there exists little to no correlation between the bulk porosity content and the reduction in tensile properties, the ductility and tensile strength are reported to decrease monotonically with an increase in the areal fraction of porosity observed on the fracture surface $[1,2,15-18]$. Accordingly, several researchers have attempted to relate the failure of die-cast metals to some measure of the areal fraction of porosity; whether determined by non-destructive evaluation or by coupling quantitative fractography with extreme value statistics [1,2,18-22].

A tensile failure model, first presented by Ghosh and later developed by Cáceres and Selling, approximates the effects of multiple voids $\left(A_{i}\right)$ as a single geometric imperfection of equivalent area $\left(A_{v}=\sum A_{i}\right)$, located at the centre of an otherwise perfect specimen $[1,2,23]$. The geometry assumed in this model is illustrated in Fig.1(i). Under uniaxial tension, the defective region yields first as a consequence of the reduced load-bearing area. The subsequent localisation of plastic flow results in the formation of an incipient neck around the local inhomogeneity. Following Ghosh's model for the loss of mechanical stability in a deforming body, the rate of strain concentration can be described considering the strain hardening ability of the material [23]. For a sample of initial cross-sectional area $A_{o}$ containing an imperfection of areal fraction $f=A_{v} / A_{o}$, axial load equilibrium requires:

$$
\sigma_{v}(1-f) A_{o} \exp \left(-\varepsilon_{v}\right)=\sigma_{h} A_{o} \exp \left(-\varepsilon_{h}\right)
$$

Where $\sigma_{v}, \varepsilon_{v}$ and $\sigma_{h}, \varepsilon_{h}$ are the true stresses and true strains in the void containing region and in the homogeneous material respectively. By coupling equation Eq.(1) with the following constitutive equation, we may solve for $\varepsilon_{h}$ when $\varepsilon_{v}$ reaches some critical fracture strain $\varepsilon_{v}^{*}$ :

$$
\sigma=K \varepsilon^{\eta}
$$

where $K$ is a material constant, $\sigma$ is the true stress, $\varepsilon$ is the true strain, and $\eta$ is the strainhardening exponent of the material. Substituting Eq.(2) into Eq.(1), we may relate the strain in the defective region to the homogeneous strain:

$$
\varepsilon_{v}^{\eta}(1-f) \exp \left(-\varepsilon_{v}\right)=\varepsilon_{h}^{\eta} \exp \left(-\varepsilon_{h}\right)
$$

Solving Eq.(3) allows the ductility of the sample to be determined by plotting $\varepsilon_{v}$ against $\varepsilon_{h}$ and noting the homogeneous fracture strain $\varepsilon_{h}^{*}$ attained when the ordinate reaches some critical fracture strain $\varepsilon_{v}^{*}$, as illustrated in Fig.1(ii). The tensile strength of the material $\sigma_{f}$ may then be determined using Eq. (4), where $\sigma^{*}$ is the true fracture strength of the 'defect-free' material obtained from $\sigma^{*}=K \eta^{\eta}$, corresponding to Considéres criterion $\varepsilon=\eta$.

$$
\sigma_{f}=\sigma^{*}\left[\frac{\varepsilon_{h}^{*}}{\eta}\right]^{\eta}
$$

Clearly, the predicted fracture strain depends on the estimated values of $f, \eta$ and $\varepsilon_{v}^{*}$. Intuitively speaking, the predicted fracture strain depends on (i) the method used to characterise defects in the material, and (ii) the method used to describe the response of the material to plastic deformation. With regards to the former, an accurate measure of $f$ can easily be obtained from the fracture surface of post-mortem specimens. However, when the location of fracture is unknown (i.e. prior to deformation) the estimation of $f$ is not so trivial. The problem now becomes that of predicting which defects will be involved during tensile failure, and estimating 
the resultant value of $f$. By acquiring a non-destructive measure of $f$ using X-ray tomography, and using this as input for the critical local strain model, Weiler et al. [24] were able to predict the location of fracture, the tensile fracture strain and the tensile fracture stress to within $8 \%$, $22 \%$, and $11 \%$ error respectively. It is possible that this relatively high error stems from the axiom that fracture will occur on the tomographic plane containing the maximum dimension of the largest defect. By considering the 3D volume as a sequence of independent $2 \mathrm{D}$ images, the authors effectively constrain the fracture path to a single elevation. Whilst this assumption might be accurate for defects which are highly spherical and/or small in comparison to the casting dimension, this is not the usually the case.

The purpose of this contribution is to present a new characterisation technique, which identifies which defects will be involved during tensile failure and captures their true 3D form. By alleviating the spatial constraints of its predecessors, the proposed technique is capable of estimating $f$ with utmost precision. The practical implications of this improvement are demonstrated by coupling the proposed technique with the critical local strain model to predict the tensile properties of Al-Si alloy die-castings containing natural casting defects.
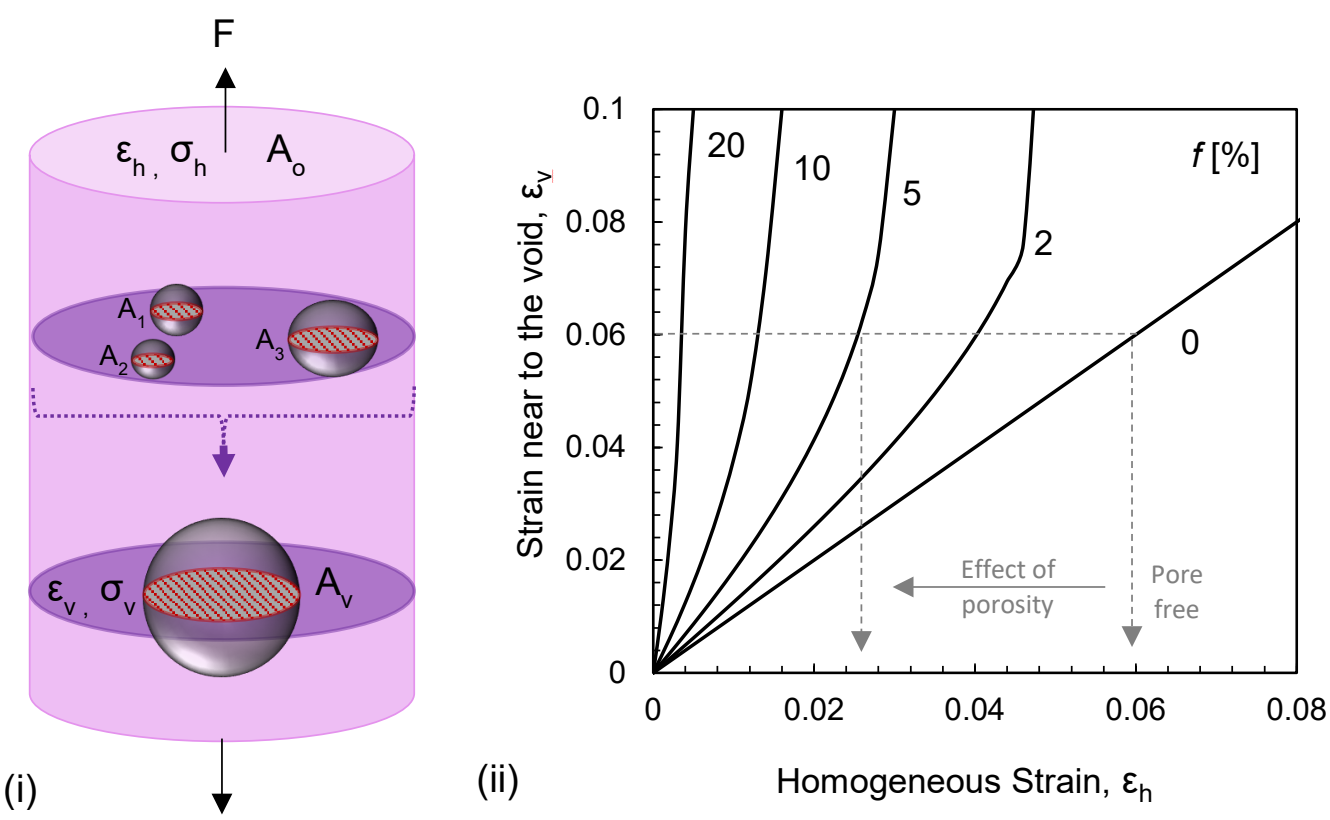

Fig.1.(i) Geometry assumed by the critical local strain model, in which the effects of multiple voids of area $A_{1}, A_{2}, \ldots A_{n}$ are approximated by a single spherical void of area $A_{v}=\sum A_{i}$ located at the centre of an otherwise perfect specimen. (ii) Curves derived from Eq.(3) showing the relation between strain in the void containing region and the homogeneous strain for different areal fractions of porosity (f). 


\section{Experimental Method}

\subsection{Specimen Preparation}

A typical AlSiMnMg die-casting aluminium alloy ( $8 \% \mathrm{Si}, 0.4 \% \mathrm{Mn}, 0.3 \% \mathrm{Mg}$ ) was used as a base material. The melt was prepared in a clay-graphite crucible (30 Kg charge) in an electric resistance furnace at $700{ }^{\circ} \mathrm{C}$. The melt was degassed using a conventional rotary degassing unit ( $5 \mathrm{~L} / \mathrm{min}$ at $350 \mathrm{rpm}$ for $10 \mathrm{~min}$ ) and then manually poured at $680^{\circ} \mathrm{C}$ into an ASTM B108 standard permanent steel mould [25], pre-heated to $460^{\circ} \mathrm{C}$. In total, 16 tensile specimens were produced, with dimensions shown in Fig.2(i). The geometry of the casting mould in addition to the nominal flow of liquid metal is provided in Fig.2(ii). To acquire the strainhardening exponent $\eta$ and strength coefficient $K$ required in Eq.(2), a 'pore-free' sample of dimensions equivalent to Fig.2(i) was machined from the bottom region of a custom made gravity die-casting shown in Fig.2(iii); Fig.2(iii) depicts a prediction of shrinkage porosity in the 'pore-free' casting calculated using the POROS 1 module based on ProCAST code [26,27].

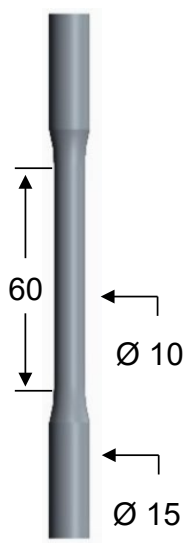

(i)

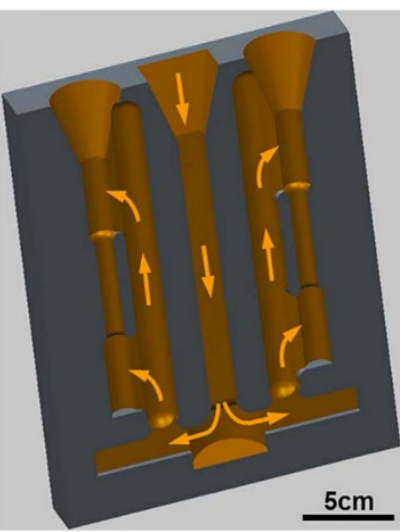

(ii)
Total Shrinkage Porosity [\%]

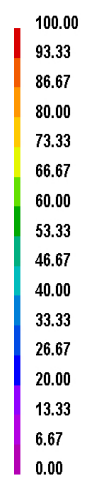

(iii)

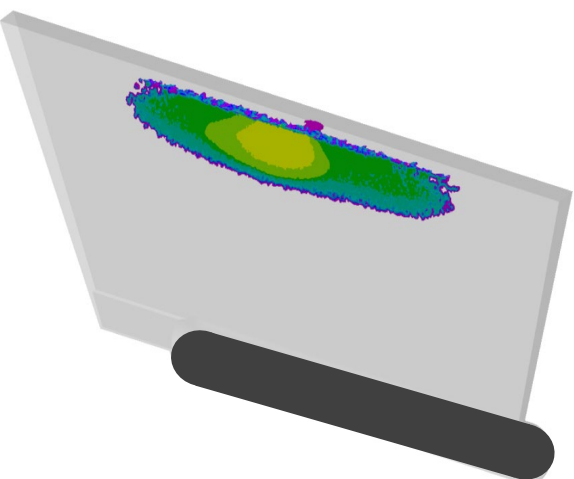

Fig.2. (i) Specimen geometry (ASTM B108 [25]). (ii) Geometry of the mould used to produce tensile specimens (arrows indicate the nominal flow of liquid metal). (iii) Prediction of shrinkage porosity within the casting used to produce the 'pore free' sample. Simulations were performed using the POROS1 module based on ProCAST code.

\subsection{X-ray Tomography and 3D Analysis}

X-ray inspection was carried out using a Y.CT Compact XL Mag computed tomography system (YXLON International, 450KV, 1mA), for which data acquisition and tomographic reconstructions are performed in parallel. An anisotropic voxel was defined, corresponding to a 2D pixel size of $133 \mu \mathrm{m}$ and an interstitial spacing of $200 \mu \mathrm{m}$. The VGSTUDIO MAX software suite (Volume Graphics Inc.) was used to visualise and analyse the 3D volumes. An iterative local surface determination was performed to define outer boundaries of the material volume whilst compensating for local fluctuations in grey value. Pore descriptors (relating to size, shape and location) were obtained using the VGDEFX void and inclusion analysis module by Volume Graphics Inc. 


\subsubsection{Predicting the location of fracture}

To identify a suitable size descriptor, two methods were used to predict the location of tensile fracture (defined here as the longitudinal distance from the bottom of the gauge section) and then compared to the actual location of fracture. In the first method, the location of fracture was predicted to occur on the tomographic plane corresponding to the centroid of the pore with the largest Feret diameter (Diameter). The second method utilizes a similar approach to that of Weiler et al. [24], in which fracture was assumed to occur on the tomographic plane containing the maximum projected area of porosity in the tensile direction (Proj. Area). A visualisation of these two methods is shown in Fig.3(i).

\subsubsection{Predicting the areal fraction of porosity involved in tensile failure}

The Diameter and Proj. Area methods may also be used to estimate the areal fraction of porosity $f_{\text {Diameter }}$ and $f_{\text {Proj.Area }}$ involved during failure: by evaluating $f$ on the transverse plane coincident with the corresponding fracture locations (section 2.2.1). However, considering the inherently complex morphology of an interconnected shrinkage pore, it seems unreasonable to assume that its maximum projected area will lie exactly on one of the tomographic planes. Hence, acquiring a measure of $f$ using the 2D images alone will likely result in an underestimate of the true value.

To overcome these spatial constraints, a new estimator (Z-Project) is presented, in which the series of images within a specified range are superimposed to estimate the maximum value of $f$ within the 3D sub-volume (Fig.3(ii)). To achieve this, each tomographic dataset is imported as an image sequence into the Fiji image post-processing software based on ImageJ [28]. The tomographic plane containing the maximum projected area of porosity is then defined as a starting position for the subsequent superposition (i.e. Proj. Area). From this plane, the sequence of images within a search distance of $\pm \sqrt{\text { area }} / 2$ are examined to identify extensions of the projection in the transverse plane of the main defect along the principle stress axis; where $\sqrt{\text { area }}$ (square root of the area of the defect projected in the direction of principle stress) is selected as a representative dimension of length due to its relation with the maximum stress intensity factor along a 3D crack (i.e. $\left.K_{I_{\max }} \propto(\sqrt{\text { area }})^{1 / 2}\right)[29,30]$. The minimum intensity of each pixel within this sub-sequence is then superimposed onto a single plane and the areal fraction of porosity measured from the generated image $\left(f_{\text {z-project }}\right)$. 

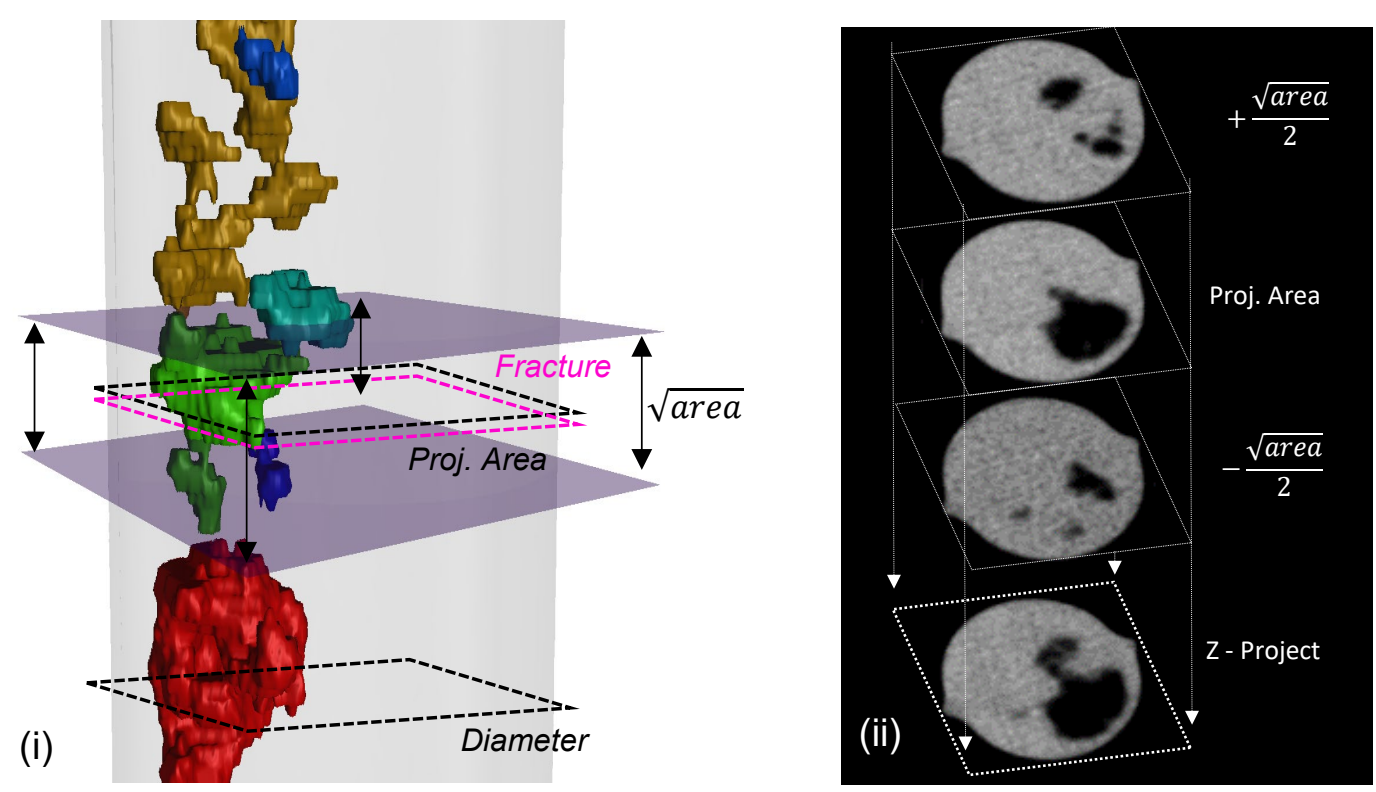

Fig.3. (i) 3D visualisation of internal porosity within the tensile specimen, showing the location of fracture predicted using the Diameter and Proj. Area methods respectively. (ii) Overview of Z-Project methodology, highlighting the starting position (Proj. Area) in addition to the upper and lower limits of the image superposition.

\subsection{Mechanical properties and fracture characterization}

Following X-ray inspection, tensile tests were performed at room temperature using an Instron 5500 universal electromechanical testing system equipped with a $50 \mathrm{kN}$ load cell, in accordance with ASTM standard E8/E8M [31]. Post-mortem tensile specimens were examined to identify the true location of fracture, with the areal fraction of porosity on the fracture surface obtained via quantitative fractography. The location of fracture was determined by the average distance from the bottom of the gauge section to the plane defined by several symmetric points along the lateral fracture surface, in a similar manner to that described in references [32,33].

\subsection{Critical local strain model}

To describe the strain-hardening behaviour of the material, values of $K$ and $\eta$ were acquired from the true stress-strain curve of the 'pore-free' sample. Eq.(3) was then solved numerically in Matlab (Mathworks Inc.) and the homogenous strain $\varepsilon_{h}$ plotted against the strain in the void containing region $\varepsilon_{v}$. A critical fracture strain $\varepsilon_{v}^{*}$ was defined to denote failure in the void containing region, as determined by the ductility of the 'pore-free' sample (i.e. when $f=$ $\left.0, \varepsilon_{v}^{*}=\varepsilon_{h}^{*}\right)$. For $f \neq 0$, strain concentrates in the void containing region and $\varepsilon_{v}^{*}>\varepsilon_{h}^{*}$. In this case, the ductility of the sample is determined by the value of $\varepsilon_{h}$ attained when $\varepsilon_{v}=\varepsilon_{v}^{*}$. This concept is illustrated by the dashed lines in Fig.1(ii) for the particular case $\varepsilon_{v}^{*}=0.06$ and $f=$ 0.05 .

\section{Results}

\subsection{Characterisation of internal porosity}

Fig.4 depicts a 3D visualisation of porosity within the gauge length of the tensile specimens obtained via X-ray tomography. In agreement with the underlying assumption of the critical local strain model, fracture is likely to be dominated by the presence of a single macropore or 
a condensed cluster of pores, covering in both cases a sizeable fraction of the cross-sectional area. Pore morphology has also been found to play an important role in the distribution of strain. Stress concentrations induced by shrinkage cavities of an interconnected and tortuous nature have proven to be more severe than those of near-spherical gas pores of an equivalent size $[9,11,34]$. Sphericity, which relates the surface area of a particle or void to the surface area of a perfect sphere of equivalent volume, is commonly used to characterise the morphology of a particle or void [7,35]. In Fig.5, the sphericity of each pore is plotted against its corresponding maximum Feret diameter. Pores of sphericity greater than 0.4 were assumed to originate from gaseous sources, whilst those of sphericity below 0.4 were attributed to solidification shrinkage or oxide films. From the 648 pores analysed by the VGDEFX algorithm, a quasilogarithmic relationship was obtained between the sphericity and maximum Feret diameter.

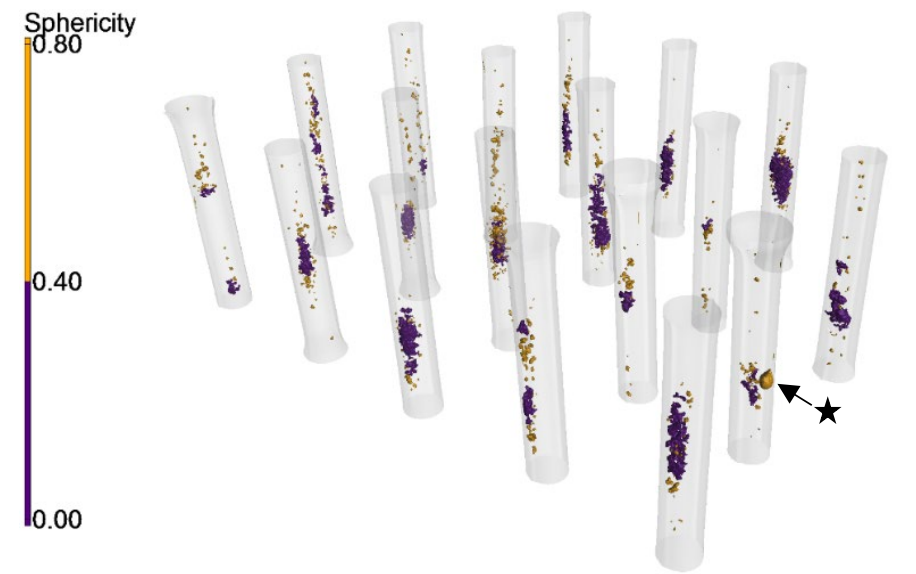

Fig.4. 3D visualisation of porosity within the gauge length of tensile specimens obtained via $\mathrm{X}$-ray tomography. Each connected pore is designated a colour based on its sphericity. Pores of sphericity greater than 0.4 were believed to originate from gaseous sources, whilst those of sphericity below 0.4 were attributed to solidification shrinkage or oxide films.

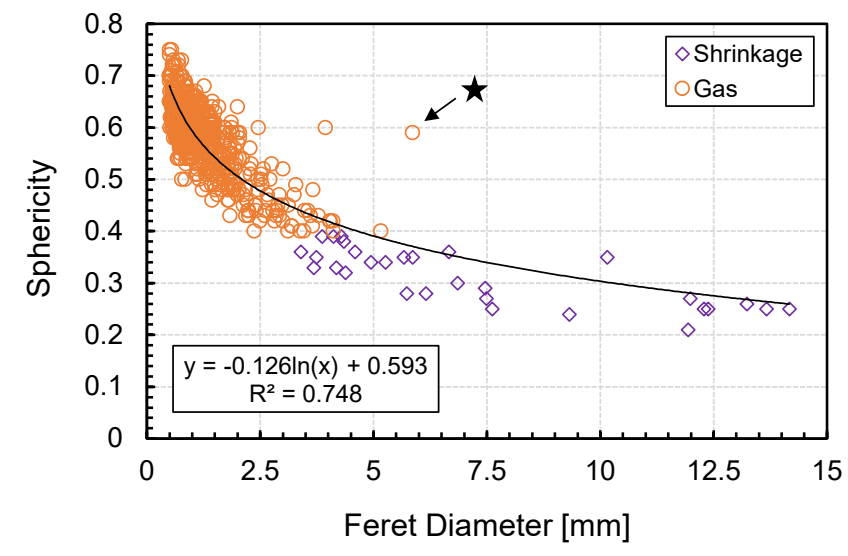

Fig.5. Relationship between sphericity and maximum Feret diameter. Pores of sphericity less than 0.4 are attributed to solidification shrinkage or oxide films, whilst those of sphericity greater than or equal to 0.4 are believed to originate from gaseous sources. 


\subsection{Fracture location}

In Fig. 6 the fracture locations predicted using the Diameter and Proj. Area approaches are plotted against the actual location of fracture measured after tensile testing. Least squares linear regression fittings are also included in the graph, with the corresponding coefficient of determination $\left(\mathrm{R}^{2}\right)$ provided for each method. Predictions based on the Proj. Area method clearly surpass their Diameter method counterparts, with average errors of 5\% (max. 18\%) and $15 \%$ (max. $75 \%$ ) reported for each method respectively. It follows then, that the maximum projected area of a pore in the tensile direction is a superior size descriptor than the maximum Feret diameter. An example of this difference is also shown in Fig.3(i), for which failure was observed to occur not at the pore with the largest Feret diameter but instead at a smaller pore with a larger projected area in the transverse plane. These findings are in strong agreement with previous reports describing a quasi-linear relationship between the tensile fracture strain and the projected area of the largest defect observed on the fracture surface $[16,17]$.

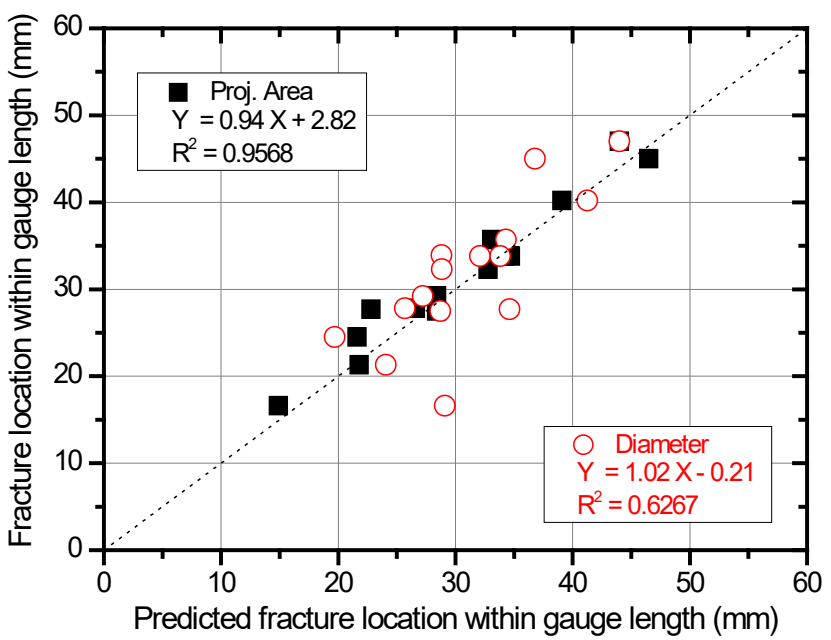

Fig.6. Comparison between the true fracture location and those predicted using the Diameter and Proj. Area methods respectively. Linear fittings and corresponding $\mathrm{R}^{2}$ coefficients are also included to compare the predictability of both methods.

\subsection{Areal fraction of porosity}

Fig.7(i,ii,iii) presents a visual representation of the areal fraction of porosity estimated using the Diameter, Proj. Area, and Z-Project methods respectively, with the actual fracture surface shown in Fig.7(iv). One might expect predictions based on the Diameter approach to highly depend on pore morphology, as the maximum Feret diameter considers only the largest dimension of the defect without regards to the principle stress axis. Accordingly, the image produced using the Diameter approach is shown to drastically underestimate the true areal fraction of porosity. Whilst there are some distinct similarities between the image produced using the Proj. Area method and the actual fracture surface, it is evident that the true fracture path transcends the tomographic plane and traverses multiple elevations. In contrast to this, the Z-Project method provides an excellent measure of the areal fraction of porosity, with the superimposed image closely resembling the actual fracture surface. Fig. 8 presents a quantitative comparison between the values of $f$ estimated using the three prediction methods and the true areal fraction of porosity measured from fracture surface. For the majority of samples, the Diameter and Proj. Area methods produce an underestimate of $f$. The 
only exception to this was the value of $f$ estimated for Sample 4 (marked with a star symbol in Figures 4, 5 and 8) using the Proj. Area method, for which failure was observed to occur at a highly spherical gas pore.

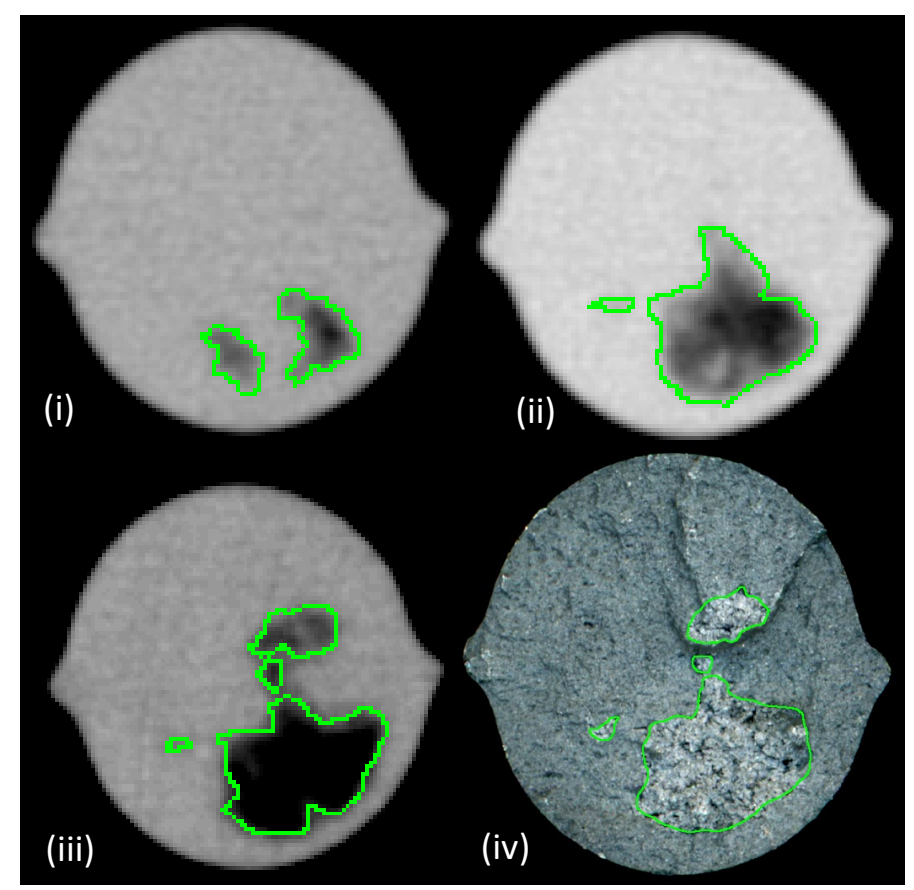

Fig.7. Areal fraction of porosity estimated for a representative sample using the (i) Diameter, (ii) Proj. Area, and (iii) Z-Project methods respectively. For comparison, the actual fracture surface is provided in (iv). Here, green lines denote the outer boundaries of each defect.

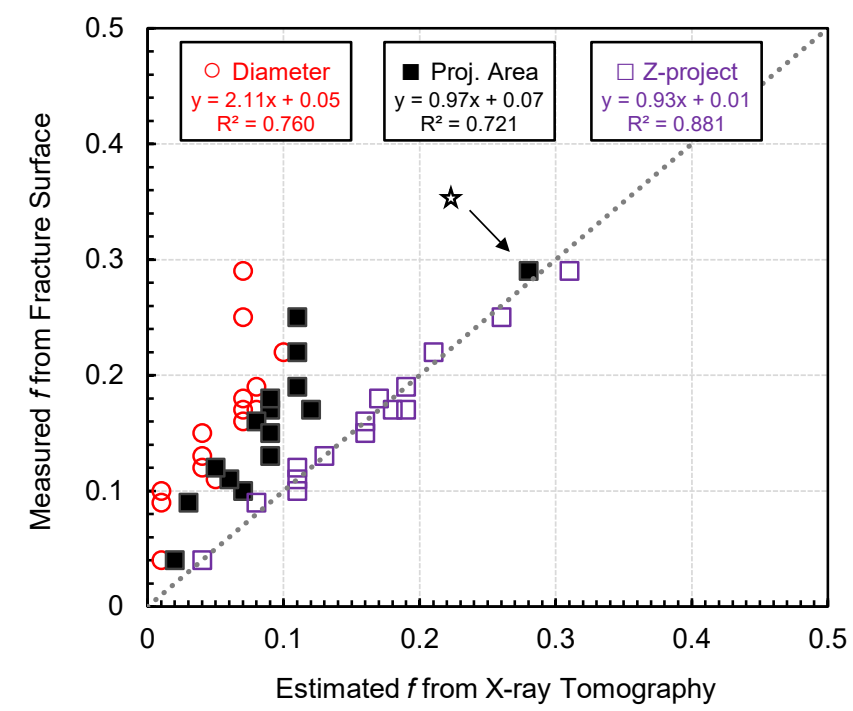

Fig.8. Comparison between the true areal fraction of porosity, $f$, and those estimated using the Diameter, Proj. Area, and Z-Project methods respectively. 


\subsection{Mechanical Properties}

The measured tensile properties of the die-cast Al8SiMnMg alloy samples are shown in Fig.9(i). The $0.2 \%$ proof strength appears to be relatively unaffected by changes in porosity, with an average value of $101.1 \pm 4.8 \mathrm{MPa}$. In contrast to this, a high degree of variability is observed for the tensile strength and the tensile ductility, with average values of $175.1 \pm 20.2 \mathrm{MPa}$ and 2.35 $\pm 1.12 \%$ respectively. This relatively high variability in mechanical properties results from variations in the size and spatial distribution of casting defects within each sample, as will be discussed later on in this paper. A power law relation was observed between the ductility and tensile strength, as would be expected from a material which exhibits strain-hardening behaviour.

Conventionally, the parameters $K, \eta$ and $\varepsilon_{i}^{*}$ are derived from the maximum fracture strain recorded in a large series of tensile tests. The assumption is that for a sufficiently large sample size (e.g. 1000 samples [24]), the maximum strain is likely to approach that of a defect free material. However, this induces significant cost and should be avoided when possible. In this study, we opt to obtain values of $K, \eta$ and $\varepsilon_{v}^{*}$ from a single 'pore-free' specimen machined from the custom made gravity die-casting shown in Fig.2(iii). X-ray tomography confirmed that no pores could be detected inside the machined specimen. Shown in Fig.9(ii) is the true stressstrain curve used to obtain the strength coefficient $K$ and strain-hardening exponent $\eta$ of the 'pore-free' material $(f=0)$. Using this approach, values of $K=341$ and $\eta=0.164$ were obtained by fitting data in the plastic region to Eq.(2). For comparison, the true fracture strain and true fracture stress of the ASTM specimens $(f>0)$ are also plotted in Fig.9(ii). Clearly, the points for $f>0$ fall along the curve for $f=0$, suggesting that the strain-hardening behaviour of the material is adequately described by the 'pore-free' specimen.
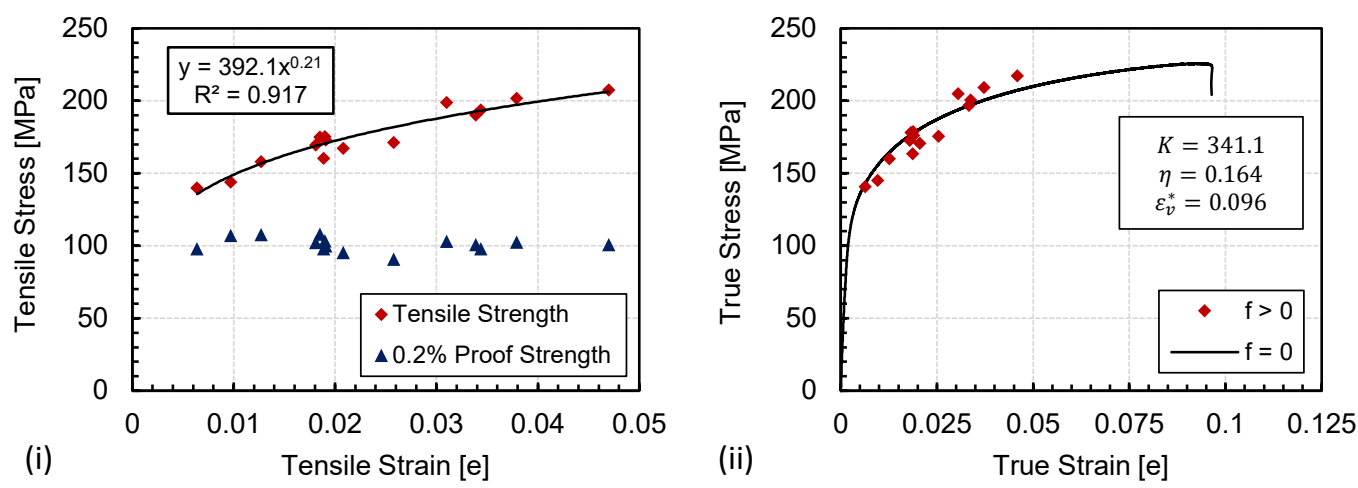

Fig. 9. (i) Tensile properties of the Al8SiMnMg alloy die-castings acquired from the engineering stress-strain curves of each sample. (ii) True stress strain curve of the 'pore-free' sample $(f=0)$ used to acquire the values of $K, \eta$ and $\varepsilon_{v}^{*}$ required of the critical local strain model. Also shown are the true fracture strain and true fracture stress for each of the tensile specimens $(f>0)$. 


\subsection{Critical local strain model}

Using the methodology outlined in section (2.4), a critical fracture strain $\varepsilon_{v}^{*}=0.096$ was defined to denote failure in the void containing region. The homogeneous fracture strain $\varepsilon_{h}^{*}$ was then determined by the value of $\varepsilon_{h}$ attained when $\varepsilon_{v}=0.096$ for a given value of $f$ : this concept is illustrated in Fig.1(ii) for the hypothetical case of $\varepsilon_{v}^{*}=0.06$ and $f=0.05$. The tensile fracture stress was then calculated for each value of $\varepsilon_{h}^{*}$ using Eq.(4).

Eq.(3) and Eq.(4) were solved numerically in Matlab for a range of $f$, with the predicted fracture strain and fracture stress plotted as a function of $f$, as shown in Fig.10(i) and Fig.10(ii) respectively. For comparison, the experimental data points obtained from the tensile tests are also included. Clearly, the critical local strain model can predict the tensile fracture strain and tensile fracture stress to a reasonable degree of accuracy, with average errors of $4.5 \%$ (max. 8.9 $\%)$ and $3.9 \%$ (max. $8.3 \%)$ respectively.
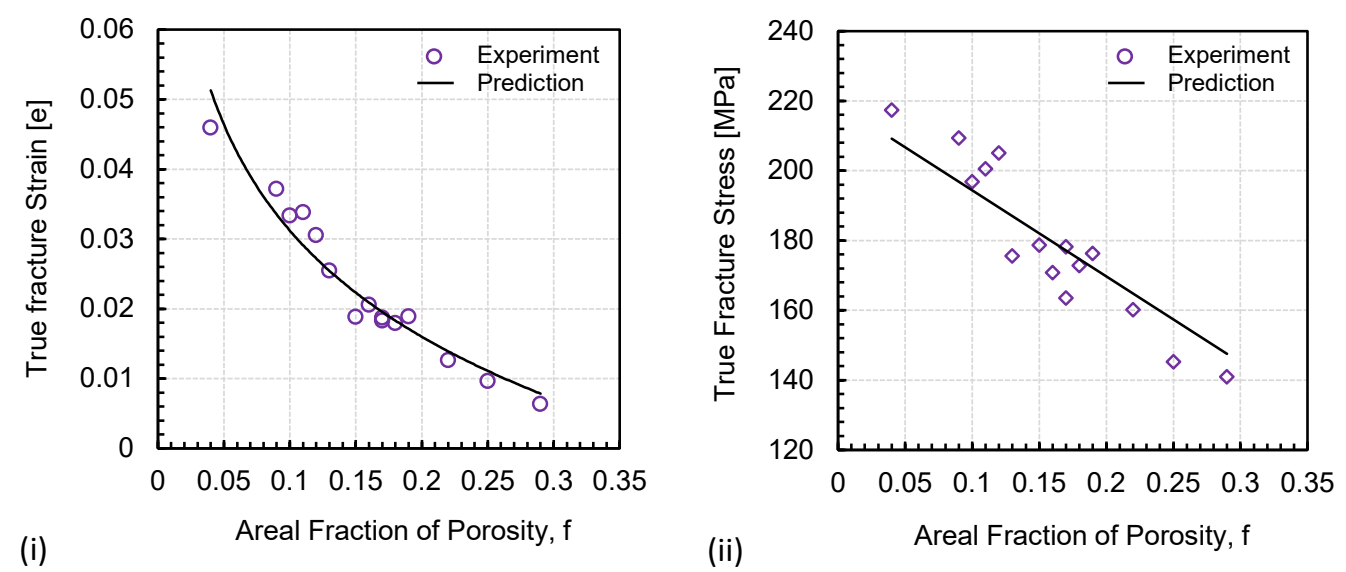

Fig.10. Comparison between the predicted and measured tensile fracture strain (i) and tensile fracture stress (ii). For the experimental data points the fracture properties obtained via tensile testing are plotted against the corresponding value of $f$ measured on the fracture surface.

\section{Discussion}

\subsection{Statistical analysis of defect size}

The extremal types theorem allows one to make inference on the probability of low-frequency high-severity events based on limited data. Methods based on the statistics of extremes are extensively used within material science and engineering to study variability in mechanical properties, particularly in modern durability (fatigue) analysis where statistical relationships are often established between the defect size distribution and the variability in fatigue life [36]. In contrast to this, such techniques are rarely used to relate the probability distribution of defect size to the variability in tensile ductility [16].

Here, the generalized extreme value (GEV) distribution is used to approximate the limiting distribution of the maxima of defect size. The GEV distribution combines the Gumbel, Fréchet and Weibull families into a single functional form, with the data themselves deciding which of the three distributions is most appropriate. The GEV distribution is parameterized by a location 
parameter $\mu$, a scale parameter $\sigma$ and a shape parameter $\xi$, which controls the tail behaviour of the distribution. The cumulative distribution function (CDF) of the GEV distribution is:

$$
G(z)=\exp \left\{-\left[1+\xi\left(\frac{z-\mu}{\sigma}\right)\right]^{-1 / \xi}\right\}, 1+\xi\left(\frac{z-\mu}{\sigma}\right)>0 .
$$

For $\xi \rightarrow 0$, the GEV distribution is equivalent to the Gumbel distribution, which is characterised by an exponentially decreasing tail:

$$
G(z)=\exp \left[-\exp \left(-\frac{z-\mu}{\sigma}\right)\right] .
$$

For $\xi>0$, the GEV distribution is equivalent to the Fréchet distribution, which possesses a polynomially decreasing tail and a finite lower bound $\mu^{\prime}$ :

$$
G(z)=\left\{\begin{array}{cc}
0, & z<\mu^{\prime} \\
\exp \left[-\left(\frac{z-\mu^{\prime}}{\sigma^{\prime}}\right)^{-1 / \xi}\right], & z \geq \mu^{\prime} .
\end{array}\right.
$$

Where we introduce two new parameters, $\mu^{\prime}$ and $\sigma^{\prime}$, for $\xi \neq 0$ :

$$
\mu^{\prime}=\mu-\frac{\sigma}{\xi}>0, \quad \sigma^{\prime}=\left|\frac{\sigma}{\xi}\right|>0 .
$$

$\xi<0$ corresponds to the Weibull distribution, which possesses a finite upper bound $\mu^{\prime}$ :

$$
G(z)=\left\{\begin{array}{cc}
\exp \left[-\left(-\frac{z-\mu^{\prime}}{\sigma^{\prime}}\right)^{-1 / \xi}\right], & z<\mu^{\prime} \\
0, & z \geq \mu^{\prime} .
\end{array}\right.
$$

Here, block maxima is the natural choice of sampling technique. In the block maxima approach, the observation period is divided into a series of sub-domains with only the maximum observation within each sub-domain considered in the analysis $\left(z_{n}=\right.$ $\left.\max \left\{X_{1}, \ldots X_{n}\right\}\right)$. Accordingly, the largest defect (max. $\sqrt{\text { area }}$ ) within each tensile sample was identified and its value of $\sqrt{\text { area }}$ used in the subsequent analyses. Within Matlab, the GEV parameters were estimated using the maximum likelihood method. The empirical CDF was then computed using the Kaplan-Meier estimator, with corresponding $95 \%$ confidence intervals evaluated using Greenwood's formula. The estimated GEV parameters along with the corresponding $95 \%$ confidence intervals are presented in Table 1. Fig.11(i) presents a visual comparison between the empirical CDF and the GEV CDF computed using the parameter estimates in Table 1. Since the entire $95 \%$ confidence interval of $\xi$ lies in the negative range, we may conclude that the limiting distribution of the maxima of defect size follows Weibull. In a similar study, Teng et al. [16] found $\xi=0$ to lie within the $95 \%$ confidence range, suggesting that the pore size distribution in an Al-Si alloy low pressure die-casting may be mathematically described by all three families. The authors reasoned that subject to physical considerations, the Weibull distribution was most appropriate due to its finite upper bound $\mu^{\prime}$. Whilst defects in this study were characterised using X-ray tomography, the pore size distribution studied by 
Teng et al. [16] was acquired from 2D images of the fracture surface. It is possible that the observed differences in shape factor arise from this difference in measurement technique; as in the present contribution, pore size measurements were acquired prior to deformation.

Whilst the parameter estimates $(\xi, \sigma, \mu)$ provide valuable insight into the quality-of-fit and tail behaviour of the distribution, it is often estimates of the extreme quantiles that are of greatest interest when analysing block maxima data. Accordingly, the quantile function is obtained by inverting Eq.(5) for $G\left(z_{p}\right)=1-p$ :

$$
\begin{array}{ll}
z_{p}=\mu-\frac{\sigma}{\xi}\left[1-\{-\log (1-p)\}^{-\xi}\right], & \text { for } \xi \neq 0, \\
z_{p}=\mu-\sigma \log \{-\log (1-p)\}, & \text { for } \xi=0 .
\end{array}
$$

Where $z_{p}$ is the return level associated with a return period $T=1 / p$. Fig.11(ii) presents a return level plot for the block maxima of defect size. Regarding the quality-of-fit, the curve obtained for the fitted model provides a reasonable approximation of the empirical quantiles. For simplicity, the lower and upper bounds of the fitted curve were computed using the $95 \%$ confidence intervals for the parameter estimates $(\xi, \sigma, \mu)$. Here, we simply wish to illustrate the relation between $\xi$ and $z_{p}$ as $T \rightarrow \infty$. In this case, the negative estimate of $\xi$ (Weibull) results in a return level curve which exhibits concavity and asymptotes to a finite value $\mu^{\prime}$ at large $T$. Physically, this implies that the maximum defect size observed in an infinite number of tensile specimens is expected to converge towards some finite value $\mu^{\prime}$.

Table 1. Limiting distribution of the maxima of defect size. Shown are the GEV parameter estimates and corresponding $95 \%$ confidence intervals.

\begin{tabular}{ccccccc}
\hline$\xi$ - Mean & $\xi-95 \%$ Confidence Intervals & $\sigma$ & $\mu$ & $\sigma^{\prime}$ & $\mu^{\prime}$ \\
\hline-0.473 & {$[-0.843$,} & $-0.102]$ & 0.847 & 3.150 & 1.791 & 4.941 \\
\hline
\end{tabular}
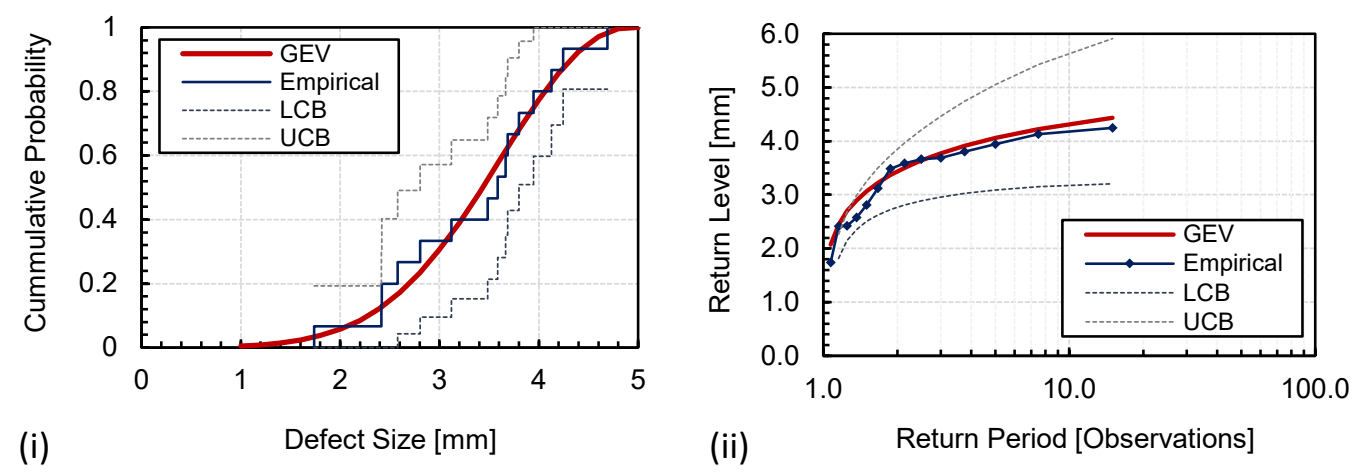

Fig.11. Limiting distribution of the maxima of defect size. (i) Comparison between the empirical CDF and the GEV CDF computed using the parameter estimates in Table 1. Here LCB and UCB refer to the lower and upper $95 \%$ confidence bounds of the empirical distribution respectively. (ii) Return level plot for the block maxima data. 


\subsection{Statistical analysis of tensile properties}

This section discusses the application of the GEV function to estimate the limiting distribution of the minima of fracture stress and fracture strain. Here, we shall focus on the probability distribution of true stress and true strain, as these are the quantities predicted by the critical local strain model. With regards to sampling, we opted to use the block minima approach. In this case, the minimum observation within each sub-domain is stored instead of the maximum value (i.e. $\tilde{z}_{n}=\min \left\{X_{1}, \ldots X_{n}\right\}$ ). A duality exists between the limiting distributions of minima and maxima such that the former may be determined by modelling the latter for $\tilde{z}_{n}=-z_{n}$ (i.e. small values of $X_{i}$ correspond to large values of $-X_{i}$ ) [37]. For the sake of computational efficiency, the GEV parameters and $95 \%$ confidence intervals were determined using the methodology outlined in Section (4.1) for $\tilde{z}_{n}=-z_{n}$, with the sign correction $\tilde{\mu}=-\mu$ (Table 2). Fig.12(i,ii) compares the empirical CDF and the GEV CDF obtained for the probability distribution of true strain and true stress respectively. Regarding the empirical distribution of true strain, the entire $95 \%$ confidence interval of $\xi$ lies in the negative range, thus we conclude that the limiting distribution of minima follows Weibull. Interestingly, $\xi=0$ lies within the $95 \%$ confidence range for the empirical distribution of true stress, suggesting that the probability distribution may be mathematically described by all three families. Following the rational of Teng et al. [16], we will discuss the physical implications of the three distributions with regards to their respective tail behaviours. If we consider the upper bounds of the $95 \%$ confidence range (i.e. $\xi>0$ ), the Fréchet distribution predicts a negative lower bound of $\tilde{\mu}=-175.1 \mathrm{MPa}$. Here, a negative threshold has no physical meaning and usually indicates a mixture of probability distributions [36]. According to Eq.(4), the diminution of tensile strength results from the reduction in tensile strain. As there is no indication of a mixed distribution for the case of tensile strain, we have opted to disregard this possibility. Furthermore, although the upper bound of $\xi$ lies in the positive range, one could equally say that the positive value $\xi=$ 0.092 in fact suggests a Gumbel distribution (i.e. $\xi \rightarrow 0$ ). Considering the return level plot in Fig.12(iv), the curve clearly exhibits some degree of concavity, again suggesting that the distribution follows Weibull. Since it is of practical interest to identify a statistical lower bound to tensile strength, the Weibull distribution is preferred to the Gumbel distribution, as the return level curve asymptotes to a finite value $\tilde{\mu}^{\prime}$. It follows then, that the presence of defects following a Weibull distribution (maxima) results in tensile fracture properties that also follow a Weibull distribution (minima): the causal relationship between the two factors is demonstrated in Fig.10, with the maximum defect size assumed to contribute to the region of $\operatorname{maximum} f$. 

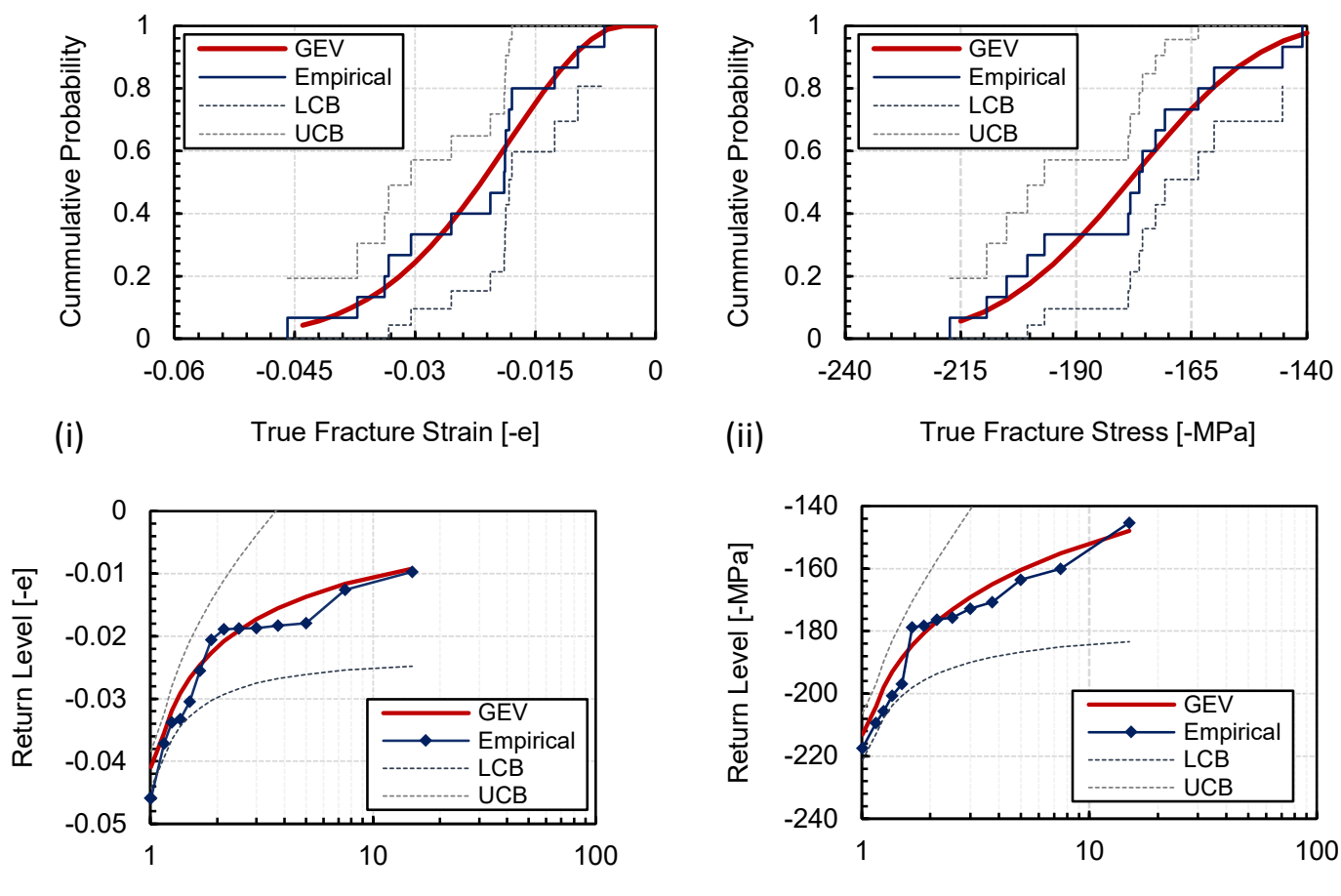

(iii) Return Period [Observations]

(iv)

Return Period [Observations]

Fig.12 Limiting distribution of the minima of true strain (i,iii) and true stress (ii,iv). Shown is a visual comparison between the empirical CDF and the GEV CDF (i,ii) and the corresponding return level plots for the block minima data (iii,iv).

Table 2. Limiting distributions of the minima of true stress and true strain. Shown are the GEV parameter estimates and corresponding $95 \%$ confidence intervals.

\begin{tabular}{|c|c|c|c|c|c|c|}
\hline & $\xi$-mean & $\xi-95 \%$ Confidence Intervals & $\sigma$ & $\tilde{\mu}$ & $\sigma^{\prime}$ & $\tilde{\mu}^{\prime}$ \\
\hline True Strain & -0.538 & {$\left[\begin{array}{ll}-0.937, & -0.138\end{array}\right]$} & 0.012 & 0.026 & 0.022 & 0.047 \\
\hline True Stress & -0.356 & {$[-0.804$,} & 22.29 & 186.4 & 62.59 & 249.0 \\
\hline
\end{tabular}

\subsection{Improved prediction of mechanical properties}

This section concerns the predictive capability of the critical local strain model when coupled with the values of $f$ estimated via X-ray tomography. Fig.13 presents a comparison between the predicted and experimental tensile fracture properties. Predictions based on the Z-Project method clearly exhibit superior accuracy with respect to the Diameter and Proj. Area methods. In Table 3, the average and maximum errors associated with the prediction of $f, \varepsilon$ and $\sigma$ are provided for the three estimators respectively. It is apparent that an improved measure of the areal fraction of porosity is accompanied by a reduction in predictive error. Accordingly, we will focus our discussion to the aptitude of the three methods in the estimation of $f$, as this controls the values predicted for the other two quantities. Here, it is useful to draw comparisons from the study of Weiler et al. [24], in which the pores analysed by the authors were much smaller and more regularly shaped $\left(f_{\max } \approx 0.06\right.$, Sphericty $\left.y_{\min } \approx 0.48\right)$ than those analysed in this study $\left(f_{\max }=0.29\right.$, Sphericty $\left.y_{\min }=0.21\right)$. It is interesting to note that, for the case 
of $\mathcal{E}$, the predictive error of the Proj. Area method observed in this study (95.2\%) is substantially larger than that reported by Weiler et al. (22\% [24]). We may infer then that for large values of $f$, the probability that the fracture plane will deviate from the transverse plane increases as the sphericity of the pore decreases. Consequently, the predictive error of conventional 2D methods (i.e. Diameter, Proj. Area) also increases with decreasing sphericity. By alleviating the aforementioned spatial constraints, the Z-Project method is capable of estimating $f$ to a superior degree of accuracy, regardless of how large or irregularly shaped these defects might be. This is confirmed by the similarity between the maximum error of the Z-Project method (fracture strain: $10.9 \%$, fracture stress: $8.9 \%$ ) and the maximum error obtained for the critical local strain model (fracture strain: $8.9 \%$, fracture stress: $8.3 \%$ ).

Despite the strong agreement between the Z-Project and baseline predictions, there is still room for improvement within the bounds of the existing model. The observed error is likely attributed to the upper and lower bounds considered for the image superposition $( \pm \sqrt{\text { area }} / 2)$. To further improve the characterization technique, a quantitative relationship shall be established between the spatial clustering of defects and the interaction of their mechanical fields. Undergoing work aims to understand this phenomenon, thus enabling a more rational upper and lower bound to be determined for the image superposition. This will further improve the accuracy of the proposed estimator.
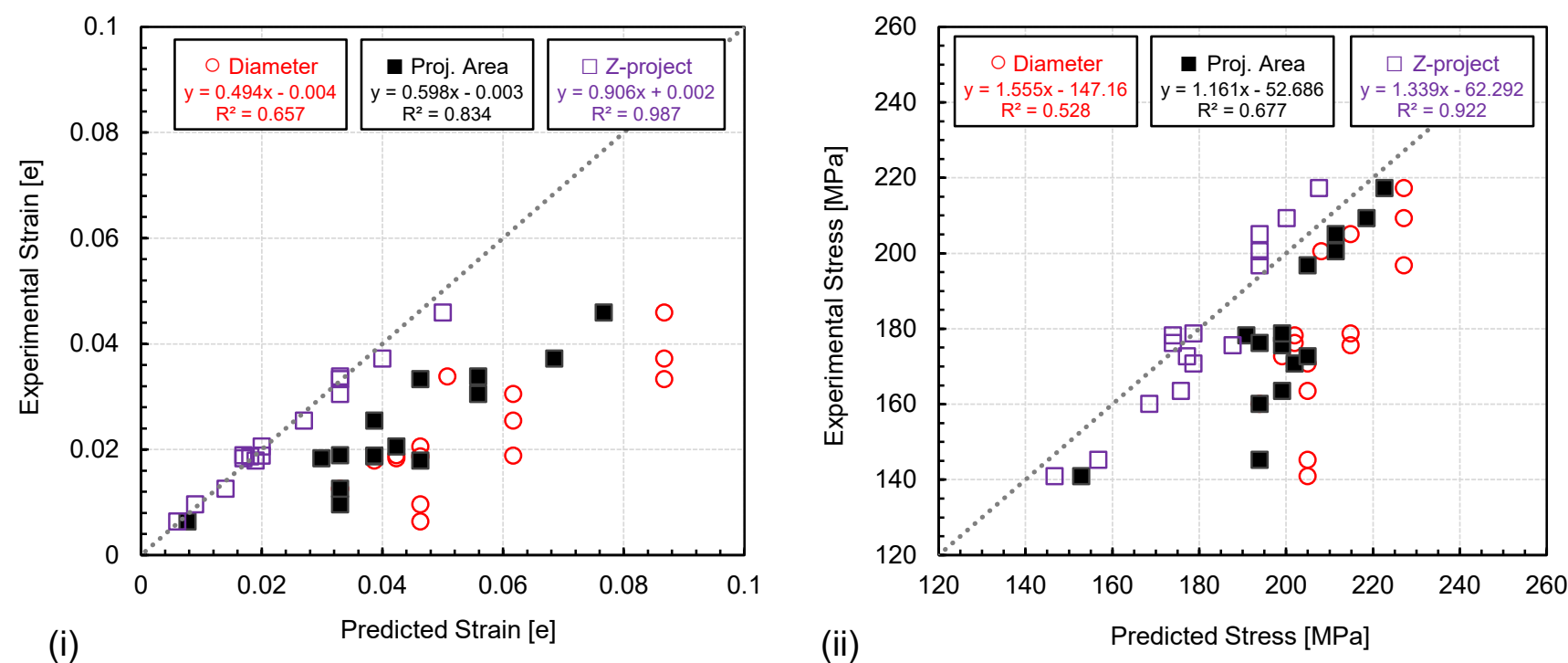

Fig.13. Tensile fracture properties predicted using the critical local strain model using values of $f$ obtained via the Diameter, Proj. Area, and Z-Project methods respectively. Here the predicted values of the (i) true fracture strain $\varepsilon$ and (ii) true fracture stress $\sigma$ are plotted against the experimental values obtained via tensile testing 
Table.3. Average and maximum (parenthesised) errors associated with the prediction of $f, \varepsilon$, and $\sigma$ for the Diameter, Proj. Area and Z-Project methods respectively.

\begin{tabular}{lccc}
\hline & \multicolumn{3}{c}{ Average Predictive error [\%] } \\
\cline { 2 - 4 } & Diameter & Proj. Area & Z-Project \\
\hline Areal fraction of porosity, $f$ & $63.3(90.0)$ & $43.3(66.7)$ & $5.00(11.8)$ \\
True fracture strain, $\varepsilon$ & $180(626)$ & $95.2(242)$ & $6.22(10.9)$ \\
True Fracture stress, $\sigma$ & $18.4(45.4)$ & $12.2(33.5)$ & $4.11(8.9)$ \\
\hline
\end{tabular}

5. Conclusions

i. A new estimator (Z-Project) is presented that can predict the areal fraction of porosity involved during tensile failure to a high degree of accuracy, far surpassing that of previous methods.

ii. By coupling the Z-Project method with an existing model for the development of plastic instabilities, the true fracture strain and true fracture stress were predicted to within $10.9 \%$ and $8.9 \%$ error, respectively. This fares extremely well against its predecessor, for which maximum errors of $242 \%$ and $33.5 \%$ were reported for the fracture strain and fracture stress, respectively. The substantial difference in error was attributed to the misalignment of the fracture plane with respect to the transverse plane, which is expected to become more severe with increasing pore size and decreasing pore sphericity. This misalignment is captured by the Z-Project estimator but is neglected in conventional approaches.

iii. The generalized extreme value function was used to model the upper tail of the defect size distribution. It was found that the limiting distribution of the maxima of defect size was best described by a Weibull distribution. It follows that the presence of defects following a Weibull distribution result in tensile fracture properties that also follow a Weibull distribution. This presents the opportunity to determine a finite upper bound for the maximum defect size, and thus a finite lower bound for the tensile fracture properties.

\section{Acknowledgements}

This work was supported by the Engineering and Physical Sciences Research Council (EPSRC) and Jaguar Land Rover Ltd. [project reference 2043200]. The authors would like to thank Dr. Feng Gao for assistance with Matlab code.

Declarations of interest: none.

The raw/processed data required to reproduce these findings cannot be shared at this time due to legal or ethical reasons.

\section{References}


[1] C.H. Cáceres, On the effect of macroporosity on the tensile properties of the Al-7\%Si$0.4 \% \mathrm{Mg}$ casting alloy, Scr. Metall. Mater. 32 (1995) 1851-1856.

[2] C.H. Cáceres, B.I. Selling, A Casting defects and the tensile properties of an Al-Si-Mg alloy, Mater. Sci. Eng. A. (1996) 109-116.

[3] C. Dørum, O.S. Hopperstad, O.-G. Lademo, M. Langseth, Aluminium and magnesium castings - Experimental work and numerical analyses, Int. J. Crashworthiness. 8 (2003) 455-470.

[4] L. Wang, N. Limodin, A. El Bartali, J.F. Witz, R. Seghir, J.Y. Buffiere, E. Charkaluk, Influence of pores on crack initiation in monotonic tensile and cyclic loadings in lost foam casting A319 alloy by using 3D in-situ analysis, Mater. Sci. Eng. A. 673 (2016) 362-372.

[5] D. Mohr, R. Treitler, Onset of fracture in high pressure die casting aluminum alloys, Eng. Fract. Mech. 75 (2008) 97-116.

[6] H. Zhang, H. Toda, H. Hara, M. Kobayashi, T. Kobayashi, D. Sugiyama, N. Kuroda, K. Uesugi, Three-dimensional visualization of the interaction between fatigue crack and micropores in an aluminum alloy using synchrotron X-ray microtomography, Metall. Mater. Trans. A Phys. Metall. Mater. Sci. 38 (2007) 1774-1785.

[7] L. Zaidao, Influence of the microstructure on mechanical properties and damage mechanisms in Al-Si-Cu alloys by using 2D and 3D in-situ analysis, Université de Lille, 2016.

[8] P. Li, P.D. Lee, D.M. Maijer, T.C. Lindley, Quantification of the interaction within defect populations on fatigue behavior in an aluminum alloy, Acta Mater. 57 (2009) 35393548.

[9] S. Dezecot, V. Maurel, J.Y. Buffiere, F. Szmytka, A. Koster, 3D characterizaation and modeling of low cycle fatigue damage mechanisms at high temperature in a cast aluminum alloy, Acta Mater. 123 (2017) 24-34.

[10] M.F. Horstemeyer, J. Lathrop, A.M. Gokhale, M. Dighe, Modeling stress state dependent damage evolution in a cast Al-Si-Mg aluminum alloy, Theor. Appl. Fract. Mech. 33 (2000) 31-47.

[11] S. Dezecot, J.Y. Buffiere, A. Koster, V. Maurel, F. Szmytka, E. Charkaluk, N. Dahdah, A. El Bartali, N. Limodin, J.F. Witz, In situ 3D characterization of high temperature fatigue damage mechanisms in a cast aluminum alloy using synchrotron X-ray tomography, Scr. Mater. 113 (2016) 254-258.

[12] J. Song, S.M. Xiong, M. Li, J. Allison, In situ observation of tensile deformation of highpressure die-cast specimens of AM50 alloy, Mater. Sci. Eng. A. 520 (2009) 197-201.

[13] I. Bacaicoa, M. Wicke, M. Luetje, F. Zeismann, A. Brueckner-Foit, A. Geisert, M. Fehlbier, Characterization of casting defects in a Fe-rich Al-Si-Cu alloy by microtomography and finite element analysis, Eng. Fract. Mech. 183 (2017) 159-169.

[14] A.M. Waters, Three-Dimensional Analysis of Voids in AMGOB Magnesium Tensile Bars Using Computed Tomography Imagery, (2001). 
[15] X. Sun, K.S. Choi, D.S. Li, Predicting the influence of pore characteristics on ductility of thin-walled high pressure die casting magnesium, Mater. Sci. Eng. A. 572 (2013) 45-55.

[16] X. Teng, H. Mae, Y. Bai, T. Wierzbicki, Pore size and fracture ductility of aluminum low pressure die casting, Eng. Fract. Mech. 76 (2009) 983-996.

[17] X. Teng, H. Mae, Y. Bai, T. Wierzbicki, Statistical analysis of ductile fracture properties of an aluminum casting, Eng. Fract. Mech. 75 (2008) 4610-4625.

[18] S.G. Lee, G.R. Patel, A.M. Gokhale, A. Sreeranganathan, M.F. Horstemeyer, Quantitative fractographic analysis of variability in the tensile ductility of high-pressure die-cast AE44 Mg-alloy, Mater. Sci. Eng. A. 427 (2006) 255-262.

[19] J.P. Weiler, J.T. Wood, Modeling fracture properties in a die-cast AM60B magnesium alloy I-Analytical failure model, Mater. Sci. Eng. A. 527 (2009) 25-31.

[20] J.P. Weiler, J.T. Wood, Modeling fracture properties in a die-cast AM60B magnesium alloy II-The effects of the size and location of porosity determined using finite element simulations, Mater. Sci. Eng. A. 527 (2009) 32-37.

[21] J.P. Weiler, J.T. Wood, Modeling the tensile failure of cast magnesium alloys, J. Alloys Compd. 537 (2012) 133-140.

[22] G. Nicoletto, G. Anzelotti, R. Konečná, X-ray computed tomography vs. metallography for pore sizing and fatigue of cast Al-alloys, Procedia Eng. 2 (2010) 547-554.

[23] A.K. Ghosh, Tensile instability and necking in materials with strain hardening and strain-rate hardening, Acta Metall. 25 (1977) 1413-1424.

[24] J.P. Weiler, J.T. Wood, R.J. Klassen, E. Maire, R. Berkmortel, G. Wang, Relationship between internal porosity and fracture strength of die-cast magnesium AM60B alloy, Mater. Sci. Eng. A. (2005) 315-322.

[25] ASTM Committee, ASTM Volume 02.02 Aluminium and Magnesium Alloys, 2006., 2006.

[26] A.J. Torroba, O. Koeser, L. Calba, L. Maestro, E. Carreño-Morelli, M. Rahimian, S. Milenkovic, I. Sabirov, J. LLorca, Investment casting of nozzle guide vanes from nickelbased superalloys: part I - thermal calibration and porosity prediction, Integr. Mater. Manuf. Innov. 3 (2014) 344-368.

[27] J. Guo, A. Makinde, B. Bewlay, Modeling of shrinkage porosity formation and its validation, Proc. from 12th Int. Conf. Model. Cast. Welding, Adv. Solidif. Process. (2009) 337-344.

[28] Fiji/ImageJ, (n.d.). https://imagej.nih.gov/ij/ (accessed August 22, 2018).

[29] Y. Murakami, M. Endo, Effects of defects inclusions and inhomogenieities on fatigue strength, 16 (1993).

[30] Y. Murakami, M. Endo, Quantitative Evaluation of Fatigue Strength of Metals Containing Various Small Defects or Cracks, Eng. Fract. Mech. 17 (1983) 1-15.

[31] ASTM committee, Standard Test Methods for Tension Testing of Metallic Materials, 2003. 
[32] Q. Xue, Y. Wang, H. Zhai, X. Chang, Automatic identification of fractures using a density-based clustering algorithm with time-spatial constraints, Energies. 11 (2018) 115.

[33] Q. Xue, Y. Wang, X. Chang, Automatic 3D fracture plane identification with spatial constraints, in: Society of Exploration Geophysicists, 2016: pp. 3443-3447.

[34] P. Mu, Y. Nadot, I. Serrano-Munoz, A. Chabod, Influence of complex defect on cast AS7G06-T6 under multiaxial fatigue loading, Eng. Fract. Mech. 123 (2014) 148-162.

[35] V. Boulos, L. Salvo, D. Houzet, P. Lhuissier, Investigating performance variations of an optimized GPU-ported granulometry algorithm, in: Des. Archit. Signal Image Process., 2012: pp. 1-6.

[36] M. Tiryakioğlu, J. Campbell, Weibull analysis of mechanical data for castings: A guide to the interpretation of probability plots, Metall. Mater. Trans. A Phys. Metall. Mater. Sci. 41 (2010) 3121-3129.

[37] S. Coles, An introduction to statistical modeling of extreme values, Springer, 2001. 